\title{
Pricing Option CGMY model
}

\author{
Manal Bouskraoui ${ }^{1}$, Aziz Arbai ${ }^{2}$ \\ ${ }^{1}$ Dept. Math. Stat. Fin., Scinece University of Abdelmalek Essaadi, Tanger - 90000, Morocco. \\ ${ }^{2}$ Dept. Math. Stat. Fin., Scinece University of Abdelmalek Essaadi, Tanger - 90000, Morocco.
}

\begin{abstract}
Empirical investigation of return dynamics leads searchers to introduce CGMY model with a particular parameter useful in characterizing the fine structure of several type of stochastic process whether the data are free or include diffusion component and whether the process contains indefinite activities and finite/in finite variation. In this paper, we summarize theoretical searcher work; this provides a CGMY-FT closed form solution algorithm for pricing option. For ac- curacy and validation we implement our method to price European call options and compare the results to a numerical simulation.
\end{abstract}

Math. Subject Classification: $60 \mathrm{H15}$

Key Words and Phrases: CGMY model, Option pricing, Levy process, Fourier transform ...

\section{Introduction}

The risk neutral approach introduced by Black \& Scholes to pricing option is 2 a paradigm in nance. Although this model remains one of the most widely used frameworks nowadays, the real prices show properties which contradict the assumptions of this model. Firstly, asset log return have been modeled in continuous time as diffusion, however, empirical studies reveal that return dynamics are devoid of diffusion component (see. Cox Ross [1976]). Secondly, asset return increments are normally distributed, yet, The distribution of price dynamics display that the increments are skewed to the left and have a fat tail than those of normal distribution (see. Fama [1963]). Finally, the implied volatility should be constant, nevertheless, it is widely recognized that the implied volatility curve reassembles a smile/skew meaning it is a convex curve of the strike price. This arguments lead to the conjecture confirmed on option data, that the risk neutral process should

- be free of diffusion component,

- model the local motion of return using both skewed and excess of kurtosis,

- count disparity phenomenon known as the volatility smile/skew.

Nowadays, recent search have been proposed CGMY process as the most suitable and ecient model to catch up assumptions fail. The model name refers to mathematician names; Carr, Geman, Madan and Yor [3] allowing to take into account both phenomenon, indefinite activity (process incorporate frequent small moves and rares large jumps) and finite/infinite variation. CGMY model has been employed to study statistical process needed to assess risk-neutral process to pricing option though the characteristic function of return price. Through this paper, we describe the ne structure of the process, then, we introduce FT technique to learn more about the distribution. Finally, we cheek whether we can dispense with diffusion so long as the process used is one of infinite activity finite variation.

\section{The CGMY model}

To obtain a clear overview of the CGMY model, we shall brie y review VG process since CGMY process extend this last by adding parameter permitting finite/infinite activity and finite/infinite variation.

\subsection{The variance Gamma process}

The idea have been displayed by Geman, Maden and Yor []. They argument that asset price return may be regarded as Brownian motion subordinated to a random clock which give rise to the name Variance Gamma process. The random clock, i.e. Gamma subordinator, is interpreted as time changed by increasing Lévy process.

Gamma subordinator with Lévy triplet $(0,0, \rho)$

Let $\left(\Omega, \mathcal{F}_{t \in[0, \infty)}, \mathbb{P}\right)$ be a filtered probability space and let $G(t ;, 1, \nu)$ be Gamma process with mean rate unity and variance $\nu$. The Lévy density of Gamma subordinator is given by

$$
\rho(x)=\frac{1}{\nu} \frac{\exp \left(-\frac{1}{\nu} x\right)}{x} 1_{x>0},
$$

following the Lévy-Khinchin representation, Laplace exponent is given by 


$$
\begin{aligned}
\mathcal{L}(\omega) & =\int_{0}^{\infty}\left(e^{\omega x}-1\right) \rho(d x), \\
& =\int_{0}^{\infty}\left(e^{\omega x}-1\right) \frac{1}{\nu} \frac{\exp \left(-\frac{1}{\nu} x\right)}{x} d x, \\
& =-\frac{1}{\nu} \ln (1-\nu \omega),
\end{aligned}
$$

since Gamma density is given by

$$
\operatorname{Gamma}_{t}(g)=\frac{\left(\frac{1}{\nu}\right)^{t / \nu}}{\Gamma(t / \nu)} g^{\frac{t}{\nu}-1} e^{-g / \nu} 1_{g>0},
$$

Variance Gamma as normal tempered 0 -stable process Let $\left(\Omega, \mathcal{F}_{t \in[0, \infty)}, \mathbb{P}\right)$ be a filtered probability space. Variance Gamma process $X_{V G}(t ; \sigma, \nu, \theta)$ with parameters $\sigma, \nu$, and $\theta$ is defined as

$$
X_{V G}(t ; \sigma, \nu, \theta)=\theta G(t ; \nu)+\sigma B_{G(t ; \nu)} .
$$

The characteristic function of Brownian motion with drift $\theta t+\sigma B_{t}$ is given by

$$
\phi_{\theta t+\sigma B_{t}}(\omega)=\exp \left\{i \theta \omega-\frac{\sigma^{2} \omega^{2}}{2}\right\},
$$

Characteristic function of Variance Gamma process is obtained by substituting Brownian motion characteristic exponent into Gamma subordinator Laplace exponent $G(t ; \nu)$ to get

$$
\begin{aligned}
\phi_{V G}(w) & =\exp \left\{t \mathcal{L}\left(\psi_{\theta t+\sigma B_{t}}(\omega)\right)\right\}, \\
& =\exp \left[t\left\{-\frac{1}{\nu} \ln \left[1+\frac{\sigma^{2} \omega^{2} \nu}{2}-i \theta \nu \omega\right]\right\}\right], \\
& =\left[1+\frac{\sigma^{2} \omega^{2} \nu}{2}-i \theta \nu \omega\right]^{-\frac{t}{\nu}},
\end{aligned}
$$

therefore, VG density is obtained by multiplying Gamma density in equation (3) to normal density of Brownian motion with drift $\theta t+\sigma B_{t}$

$$
V G\left(x_{t}\right)=\int_{0}^{\infty} \frac{1}{\sqrt{2 \pi \sigma^{2} g}} \exp \left\{\frac{-\left(x_{t}-\theta g\right)^{2}}{2 \sigma^{2} g}\right\} \frac{\left(\frac{1}{\nu}\right)^{t / \nu}}{\Gamma(t / \nu)} g^{\frac{t}{\nu}-1} e^{-g / \nu} d g,
$$

which leads to MCC [1998] equation,

$$
V G\left(x_{t}\right)=\frac{\sqrt{2} \exp \left(\frac{\theta}{\sigma^{2}} x_{t}\right)}{\nu^{t / \nu} \sigma \sqrt{\pi} \Gamma(t / \nu)}\left[\frac{x_{t}^{2}}{\frac{2 \sigma^{2}}{\nu}+\theta^{2}}\right]^{\frac{t}{2 \nu}-\frac{1}{4}} K_{\frac{t}{\nu}-\frac{1}{2}}\left[\frac{\sqrt{x_{t}^{2}\left(\frac{2 \sigma^{2}}{\nu}+\theta^{2}\right)}}{\sigma^{2}}\right],
$$

$K$ is a modified Bessel function of the second kind.

The necessity to extend Variance Gamma due to the fact that the process deals only with the case infinite activity and finite variation. 


\subsection{The CGMY process}

Contrary to Variance Gamma process represented as time changed Brownian motion, CGMY process is unknown through such representation, it is only known by its Lévy measure.

Let $\left(\Omega, \mathcal{F}_{t \in[0, \infty)}, \mathbb{P}\right)$ be a filtered probability space, we define CGMY process $X_{C G M Y}(t ; C, G, M, Y)$ as Lévy process with Lévy triplet $\left(A^{x}=0, \rho^{x}, b^{x}=0\right)$ and measure

$$
\rho_{C G M Y}^{x}=\frac{C \exp ^{(-G|x|)}}{|x|^{1+Y}} 1_{x<0}+\frac{C \exp ^{(-M x)}}{x^{1+Y}} 1_{x>0},
$$

where $C>0, G \geq 0, M \geq 0$ and $Y<2$. The parameter $C$ controls overall arrival rate of jumps, i.e. the larger value of $C$ result in the larger overall arrival rate of jumps. $G$ and $M$ are the exponential decay rates on the right and left of the Lévy measure leading to skewed distribution when they are unequal, when $G=M$, the lévy measure is symmetric. For $G<M$, the tail of $X_{t}$ distribution is heavier than the right tail, we say that the arrival rate of negative jumps is higher than that of large jumps. The most interesting parameter is $Y$, it was studied by Vershik and Yor [11], it allows to understand the structure of process since it describe the behavior of Lévy measure whether is completely monotone, has finite/infinite activity and finite/infinite variation. Table below summarizes the role of the parameter $Y$.

\begin{tabular}{|c|c|c|c|}
\hline $\mathrm{Y}$ values & \multicolumn{2}{|c|}{ Structure of CGMY process } & Lévy process \\
\hline $\mathrm{Y}$ & $\begin{array}{l}\text { Description of the pro- } \\
\text { cess CGMY }\end{array}$ & Measure $\rho_{C G M Y}$ & Lévy process \\
\hline$Y<0$ & $\begin{array}{l}\text { Finite number of jumps } \\
\text { in any finite time pe- } \\
\text { riod. }\end{array}$ & $\begin{array}{l}\text { Completely } \\
\text { monotone } \\
(-1,0)\end{array}$ & $\begin{array}{l}\text { Compound Pois- } \\
\text { son process. }\end{array}$ \\
\hline$Y \in(0,1)$ & $\begin{array}{l}\text { Process exhibits infi- } \\
\text { nite activity between } \\
\text { big jumps and finite } \\
\text { variation. }\end{array}$ & $\begin{array}{l}\text { Completely } \\
\text { monotone. }\end{array}$ & CGMY process. \\
\hline$Y \in(1,2)$ & $\begin{array}{l}\text { Process has trajectories } \\
\text { of infinite activity and } \\
\text { finite variation. }\end{array}$ & $\begin{array}{l}\text { Completely } \\
\text { monotone. }\end{array}$ & $\begin{array}{l}\text { Subordinated } \\
\text { Brownian motion. }\end{array}$ \\
\hline
\end{tabular}

The table Based on the Table [3]

the illustration below show Lévy measure behavior with various values for $G$ and $M$

As we show before, CGMY Lévy measure is the only information we dispose to identify structure of the process, since we are interested to decrypt distribution behavior, we introduce characteristic function through this theorem.

Theorem 1 (CGMY characteristic function). Let $\left(\Omega, \mathcal{F}_{t \in[0, \infty)}, \mathcal{P}\right)$ be a filtered probability space, characteristic function for infinitely divisible process $X_{t \in[0, \infty)}$ with independent increments and Lévy density is given by equa- 


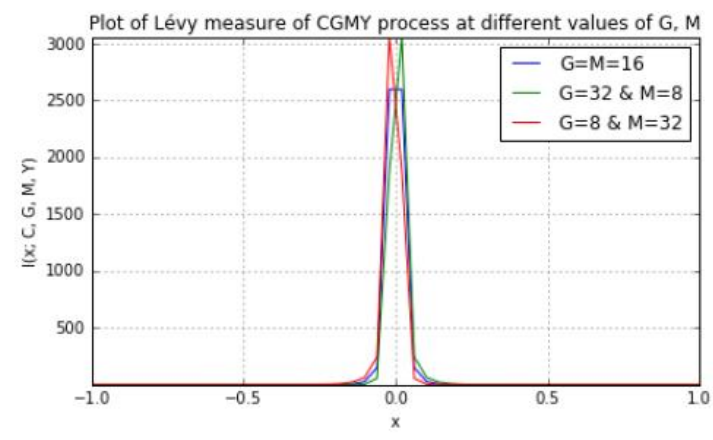

Figure 1: Parameters fixed are $C=1.2$ and $Y=1.0$

tion (8)

$$
\begin{aligned}
\phi_{C G M Y}(\omega, t ; C, G, M, Y) & =\exp \left\{t C G^{Y} \Gamma(-Y)\left[\left(1+\frac{i \omega}{G}\right)^{Y}-1-\frac{i \omega Y}{G}\right]\right. \\
& \left.+t C M^{Y} \Gamma(-Y)\left[\left(1-\frac{i \omega}{M}\right)^{Y}-1+\frac{i \omega Y}{M}\right]\right\} .
\end{aligned}
$$

Proof. Characteristic exponent of CGMY process with characteristic triplet $\left(A^{x}=0, \rho^{X}, b^{x}=0\right)$ is obtained by using Lévy-Khinchin representation without truncation of large jumps

$$
\psi_{X}(\omega)=\int_{-\infty}^{\infty}\{\exp (i \omega x)-1-i \omega x\} \rho_{C G M Y}(x ; C, G, M, Y) d x
$$

first of all, we consider the positive half of Lévy measure

$$
\begin{aligned}
& \int_{0}^{\infty}\{\exp (i \omega x)-1-i \omega x\} C \frac{\exp (-M x)}{x^{1+Y}} d x=C \sum_{2}^{\infty} \frac{(i \omega)^{n}}{n !} \int_{0}^{\infty} x^{n-1-Y} e^{-M x} d x, \\
& =C \sum_{2}^{\infty} \frac{(i \omega)^{n}}{n !} M^{Y-n} \Gamma(n-Y), \\
& =C M^{Y} \Gamma(2-Y)\left\{\frac{1}{2 !}\left(\frac{i \omega}{M}\right)^{2}+\frac{2-Y}{3 !}\left(\frac{i \omega}{M}\right)^{3}+\frac{(2-Y)(3-M)}{4 !}\left(\frac{i \omega}{M}\right)^{4}+\cdots\right\},
\end{aligned}
$$

so such the expression in braces appears as the power series

$$
(1+y)^{n}=1+n y+n(n-1) \frac{y^{2}}{2 !}+\cdots
$$

then

$$
\int_{0}^{\infty}\{\exp (i \omega x)-1-i \omega x\} C \frac{\exp (-M x)}{x^{1+Y}} d x=C M^{Y} \Gamma(-Y)\left\{\left(1-\frac{i \omega}{M}\right)^{Y}-1+\frac{i \omega Y}{M}\right\} .
$$


Next, we treat at the same manner the negative half of Lévy measure, where,

$|x|=-x$.

$$
\begin{aligned}
\int_{-\infty}^{0}\{\exp (i \omega x)-1-i \omega x\} C \frac{\exp (-G|x|)}{|x|^{1+Y}} d x & =\int_{-\infty}^{0}\{\exp (-i \omega(-x))-1+i \omega(-x)\} C \frac{\exp (-G(-x))}{(-x)^{1+Y}} \\
& =\int_{0}^{\infty}\{-\exp (-i \omega x)+1-i \omega x\} C \frac{\exp (-G x)}{x^{1+Y}} d x,
\end{aligned}
$$

until interchanging integral in the positive half we have

$$
\int_{-\infty}^{0}\{\exp (i \omega x)-1-i \omega x\} C \frac{\exp (-G|x|)}{|x|^{1+Y}} d x=C G^{Y} \Gamma(-Y)\left\{\left(1+\frac{i \omega}{G}\right)^{Y}-1-\frac{i \omega Y}{G}\right\}
$$

assembling both parts of Lévy measure to obtain characteristic function of CGMY process $\phi_{X}(\omega)$ as

$$
\exp \left[t C G^{Y} \Gamma(-Y)\left\{\left(1+\frac{i \omega}{G}\right)^{Y}-1-\frac{i \omega Y}{G}\right\}+t C M^{Y} \Gamma(-Y)\left\{\left(1-\frac{i \omega}{M}\right)^{Y}-1+\frac{i \omega Y}{M}\right\}\right] .
$$

\section{European call option pricing under CGMY model}

\subsection{CGMY asset price process}

Let $\left(\Omega, \mathcal{F}_{t \in[0, T]}, \mathbb{Q}\right)$ a filtered risk neutral probability space. Asset price dynamics $S_{t \in[0, T]}$ is an exponential Lévy process $L_{t \in[0, T]}$ of the form

$$
S_{t}=S_{0} \exp L_{t},
$$

the choice of the Lévy process is the CGMY process plus a drift

$$
L_{t} \equiv\left\{(r-\beta) t+X_{C G M Y}\left(t ; C_{\mathbb{Q}}, G_{\mathbb{Q}}, M_{\mathbb{Q}}, Y_{\mathbb{Q}}\right)\right\},
$$

where $r \geq 0$ is the mean rate of return on the asset and $\beta$ is the convexity correction in CGMY model takes the next form

$$
\begin{aligned}
\beta & =\phi_{C G M Y}(-i, t ; C, G M, Y), \\
& =C_{\mathbb{Q}} G_{\mathbb{Q}}^{Y_{\mathbb{Q}}} \Gamma\left(-Y_{\mathbb{Q}}\right)\left\{\left(1+\frac{1}{G_{\mathbb{Q}}}\right)^{Y_{\mathbb{Q}}}-1-\frac{Y_{\mathbb{Q}}}{G_{\mathbb{Q}}}\right\} \\
& +C_{\mathbb{Q}} M_{\mathbb{Q}}^{Y_{\mathbb{Q}}} \Gamma\left(-Y_{\mathbb{Q}}\right)\left\{\left(1-\frac{1}{M_{\mathbb{Q}}}\right)^{Y_{\mathbb{Q}}}-1+\frac{Y_{\mathbb{Q}}}{M_{\mathbb{Q}}}\right\} .
\end{aligned}
$$

\subsection{Characteristic function formulation for solution}

In this section, we are motivated to pursue closed-form solutions to know more about process structure and pricing option under CGMY model. The risk-neutral log asset price dynamics can be obtained from the equation (14) as

$$
\ln S_{t}=\ln S_{0}+(r-\beta) t+X_{C G M Y}\left(t ; C_{\mathbb{Q}}, G_{\mathbb{Q}}, M_{\mathbb{Q}}, Y_{\mathbb{Q}}\right),
$$

since the density of the process $X_{t}$ is expressed in the equation (8) and with the rearrangement

$$
X_{t}=\ln S_{t}-\left[\ln S_{0}+(r-\beta) t\right],
$$


the characteristic function of $\log$ asset price $\ln S_{t}$ is obtained by substituting formula of the equation (8) and added the drift term $(r-\beta) t$ as

$$
\begin{aligned}
\phi_{\ln S_{t}}(\omega) & =\exp \left\{i \omega \ln S_{0}+(r-\beta) t\right\} \\
& \times \exp \left\{t C_{\mathbb{Q}} G_{\mathbb{Q}}^{Y_{\mathbb{Q}}} \Gamma\left(-Y_{\mathbb{Q}}\right)\left[\left(1+\frac{i \omega}{G_{\mathbb{Q}}}\right)^{Y_{\mathbb{Q}}}-1-\frac{i \omega Y_{\mathbb{Q}}}{G_{\mathbb{Q}}}\right]\right. \\
& \left.+t C_{\mathbb{Q}} M_{\mathbb{Q}}^{Y_{\mathbb{Q}}} \Gamma\left(-Y_{\mathbb{Q}}\right)\left[\left(1-\frac{i \omega}{M_{\mathbb{Q}}}\right)^{Y_{\mathbb{Q}}}-1+\frac{i \omega Y_{\mathbb{Q}}}{M_{\mathbb{Q}}}\right]\right\},
\end{aligned}
$$

$\beta$ is as defined in the equation (15).

Without lost of generality, let $t=T$ and $\ln S_{T} \equiv s_{T}$, then, drop $\mathbb{Q}$ we have

$$
\begin{aligned}
\phi_{T}(\omega) & =\exp \left\{i \omega s_{0}+(r-\beta) T\right\} \\
& \times \exp \left\{T C G^{Y} \Gamma(-Y)\left[\left(1+\frac{i \omega}{G}\right)^{Y}-1-\frac{i \omega Y}{G}\right]+T C M^{Y} \Gamma(-Y)\left[\left(1-\frac{i \omega}{M}\right)^{Y}-1+\frac{i \omega Y}{M}\right]\right\} .
\end{aligned}
$$

\subsection{Moments of CGMY model}

The standardized moments are computed as indicate above

$$
\begin{aligned}
E\left[X_{t}\right] & =0, \\
\operatorname{Var}\left[X_{t}\right] & =\int_{-\infty}^{\infty} x^{2} \rho_{C G M Y}(x) d x, \\
& =t C \Gamma(2-Y)\left(G^{Y-2}+M^{Y-2}\right), \\
\text { Skewness }\left[X_{t}\right] & =\int_{-\infty}^{\infty} x^{3} \rho_{C G M Y}(x) d x, \\
& =\frac{t C \Gamma(3-Y)\left(-G^{Y-3}+M^{Y-3}\right)}{\operatorname{Var}\left[X_{t}\right]^{\frac{3}{2}}}, \\
\text { Kurtosis }\left[X_{t}\right] & =\int_{-\infty}^{\infty} x^{4} \rho_{C G M Y}(x) d x, \\
& =\frac{t C \Gamma(4-Y)\left(G^{Y-4}+M^{Y-3}\right)}{\operatorname{Var}\left[X_{t}\right]^{2}} .
\end{aligned}
$$

\subsection{Fourier transform inversion}

We substitute the characteristic function in the equation (18), we obtain CGMY-FT call pricing formula

$$
C_{C G M Y}(T, K)=\frac{e^{-\alpha k}}{2 \pi} \int_{-\infty}^{\infty} e^{-i \omega k} \frac{e^{-r T} \phi_{T}(\omega-(\alpha+1) i)}{\alpha^{2}+\alpha-\omega^{2}+i(2 \alpha+1) \omega} d \omega,
$$

where $\phi_{T}(\omega)$ is as defined in equation (19)

\section{Numerical Results}

We implement the CGMY Fourier transform formula (21) with decay rates parameters $G=2.0, M=3.5$, overall arrival rate $C=0.5$ and $Y=1.34$. We consider the common parameters $S_{0}=100.0, r=0.05$. While $\omega_{n}$ (resp. $\left.k_{p}\right)$ varies from $\omega_{n}=1, . ., N$ (resp. in the range $(-b, b)$ ) and which we assume to be equal in length. Considering the maturity $T=1.0$, time grid $M=50$ and finally, simulation size $I=10000$.

We implement CGMY model in PYTHON. The illustration below shows dynamic of stock price in market free-arbitrage. 

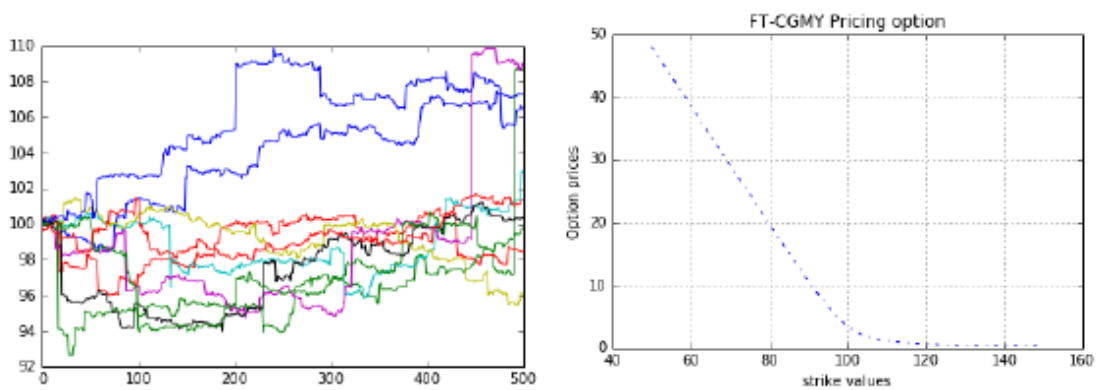

Figure 2: FT-CGMY option pricing

\section{Conclusion}

We calibrate FT-CGMY option pricing approach with the modified call price. The calibration result suggests that the extra parameters of CGMY model allow the negative skewness and the excess of kurtosis (leptokurtic). Moreover, the dynamics of implied Lévy density is asymmetric and has an infinite activity. Despite the efficiency of CGMY process, all option pricing models are biased (see. [1]). In fact, unbiased model would produce errors which are on average equal to zero. For that reason, our work is summarized in how much we could reduce the degree of misspecification. Adding jumps is the first station in this course.

\section{References}

[1] G. Bakshi, C. Cao, Z. Chen, Empirical Performance of Alternative Option Pricing Models, J. Fin., 52 (1997), 20-49.

[2] J. Bertoin, Lévy Processes, Cambridge University Press, Cambridge(1996).

[3] P. Carr, H. Geman, D. B. Madan, M. Yor, The fine structure of asset returns: An empirical investigation, J. Bus., 75 (2002), 305332.

[4] N. Cai, S. G. Kou, Z. Liu, A two-sided Laplace inversion algorithm with computable error bounds and its applications in financial engineering, Advances in Applied Probability (2013).

[5] P. Carr, H. Geman, D.B. Madan, M. Yor, Stochastic volatility for Lévy processes. J. Math. Fin., 13 (2003), 345382.

[6] R. Goldberg, Fourier Transforms. Cambridge Tracts in Mathematics and Mathematical Physics, Cambridge University Press, Cambridge (1961)

[7] R. Kawai, H. Masuda, On simulation of tempered stable random variates. J. Comp. Appl. Math., 235 (2011), 28732887.

[8] D.B, Madan, M. Yor, Representing the CGMY and Meixner Levy processes as time changed Brownian motions. J. Comp. Fin., 12 (2008), 2747 .

[9] J. Poirot, P. Tankov, Monte Carlo option pricing for tempered stable (CGMY) processes, In:Asia-Pacific Financial Markets, 13 (2006) 327344 .

[10] K. Sato, Lévy Processes and Infinitely Divisible Distributions, Cambridge University Press, Cambridge (1999).

[11] A. Vershik, M. Yor, 1995. Multiplicativité du processus gamma et étude asymptotique des lois stables d'indice a, lorsque a tend vers $Q$. Prépublication, Paris Laboratoire de Probability, Université Paris 6. 